\title{
QCD: THEORETICAL DEVELOPMENTS
}

\author{
THOMAS GEHRMANN \\ Institut für Theoretische Physik, Universität Zürich, Winterthurerstraße 190, CH-8057 Zürich, Switzerland
}

I review recent theoretical advances in quantum chromodynamics. Particular emphasis is put on developments related to the precise prediction and interpretation of experimental data from present and future high energy colliders.

\section{Introduction}

Quantum Chromodynamics (QCD) is well established as theory of strong interactions through a large number of experimental verifications. The era of 'testing QCD' is clearly finished, and QCD today is becoming precision physics. The next generation of high energy collider experiments are all performed at hadron colliders, where (in contrast to LEP and SLC) QCD is ubiquitous. Any precision measurement (strong coupling constant, quark masses, electroweak parameters, parton distributions) at the Tevatron and the LHC, as well as any prediction of new physics effects and their backgrounds, relies on the understanding of QCD effects on the observable under consideration.

The derivation of precise QCD predictions for collider observables poses several theoretical and computational challenges. The most important challenge is the fact that QCD describes quarks and gluons, while experiments observe hadrons. This mismatch is either accounted for by a description of the parton to hadron transition through fragmentation functions or by defining sufficiently inclusive final state observables, such as jets. Moreover, the strong coupling constant is considerably larger than the electromagnetic coupling constant at scales typically probed at colliders: $\alpha_{s}\left(M_{Z}\right) \simeq 15 \alpha_{\mathrm{em}}\left(M_{Z}\right)$, resulting in a slower convergence of the perturbative expansion. As a consequence, a precise description of QCD observables (precise means here that the theoretical uncertainty becomes similar to the achieved or projected experimental errors) is obtained only by including higher order corrections, often requiring beyond the next-to-leading order. The largeness of the strong coupling constant also implies that multiparticle final states are rather frequent. Finally, many collider observables involve largely different scales, such as quark masses, transverse momenta and vector boson masses. These give rise to potentially large logarithms, which might spoil the convergence of the perturbative series and need to be resummed to all orders.

In this talk, I shall try to highlight recent theoretical progress towards precision QCD at colliders, focusing on heavy quark production in Section 2, on jets and multiparton final states in Section 3, on photons in Section 4 and electroweak bosons in Section 5. Finally, a summary of the current state-of-the-art and of yet open issues is given in Section 6 .

\section{Heavy Quarks}

Heavy quark production is one of the main topics investigated at high energy collider experiments. Heavy quarks are of particular interest to probe the flavour sector of the standard model, which is less well tested than the gauge sector. Also, many approaches to physics beyond the standard model, often related to electroweak symmetry breaking and mass generation, predict new effects to be most pronounced in observables involving heavy quarks.

\subsection{Total Cross Sections}

The total cross sections for the production of heavy quarks can be computed reliably within perturbation theory. The current state-of-the-art is a nextto-leading order (NLO) calculation ${ }^{1}$, which is further improved by summing large logarithms due to soft gluon emission up to the next-to-leading (NLL) ${ }^{2}$ and next-to-next-to-leading logarithmic (NNLL) ${ }^{3}$ level. As can be seen from Figure 1, these predictions are in good agreement with experimental data on the total $t \bar{t}$ cross section at the Tevatron ${ }^{4}$ and the total $b \bar{b}$ cross section at HERA-B ${ }^{5}$ (which both actually refer to similar kinematical values of $m_{Q} / \sqrt{s}$ ). The theoretical uncertainty on the prediction for HERA$\mathrm{B}$ is larger for two reasons: the larger value of the strong coupling at $m_{b}$ than at $m_{t}$ and the dominance of $g g$ initial states in $p N$ collisions (HERA-B) compared to $q \bar{q}$ dominance in $p \bar{p}$ collisions (Tevatron). 

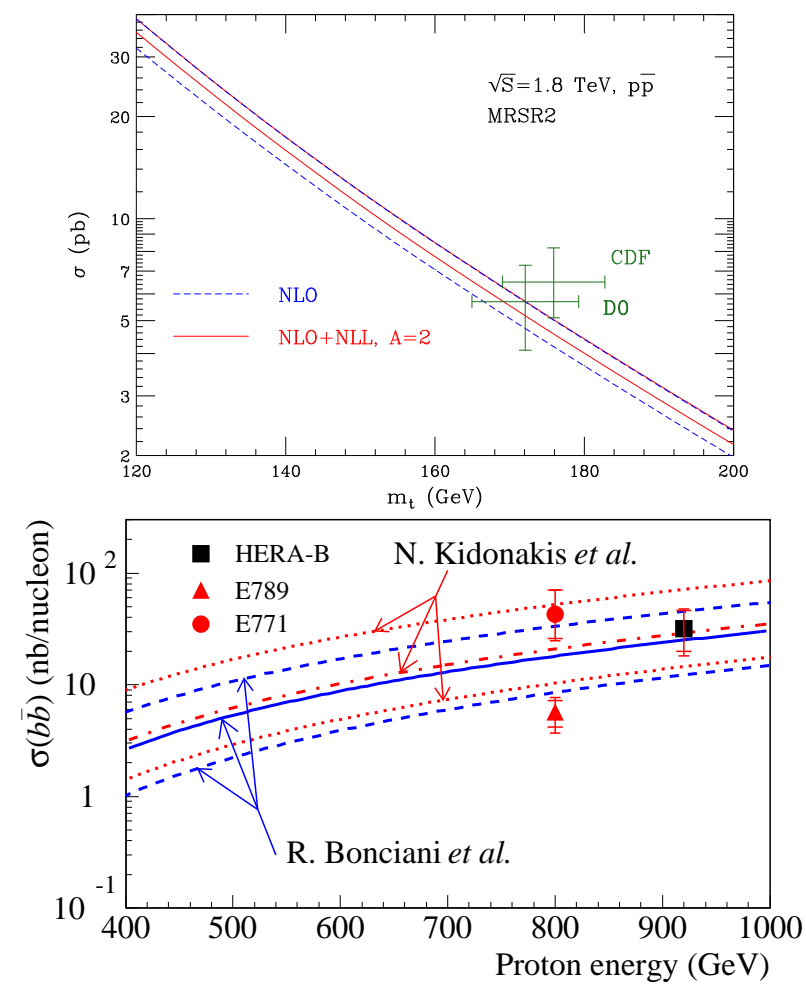

Figure 1. Total cross sections for $t \bar{t}$ at the Tevatron ${ }^{2}$ and $b \bar{b}$ at HERA-B ${ }^{5}$.

The effects due to soft gluon resummation turn out to be moderate, but do yield a significant decrease in the uncertainty of the theoretical prediction. Further uncertainties on the prediction of the $t \bar{t}$ cross section come from the parton distribution functions ${ }^{6}$.

\subsection{Transverse Momentum Distributions}

Differential distributions of hadrons containing $b$ quarks measured in hadron-hadron, photon-hadron or photon-photon collisions have been in apparent discrepancy with theoretical predictions for quite some time. The spectrum of $B^{ \pm}$hadrons measured at $\mathrm{CDF}^{7}$ is one of the most recent examples for this discrepancy.

The theoretical prediction for $B$ meson production involves a convolution of the hard matrix element for heavy quark production in parton-parton scattering with initial parton distributions and final state fragmentation functions describing the nonperturbative transition from a $b$ quark to a $B$ hadron. It is in particular the latter which is suspected to account for the discrepancy between theoretical prediction and experimental data, especially since it has been observed ${ }^{8}$ that the transverse momentum distribution of $b$-tagged jets ${ }^{9}$ (which has little sensitivity to fragmentation functions) is in much better agreement with theoretical predictions.

The definition of heavy quark fragmentation functions is not free from ambiguities, since some aspects of these functions are actually calculable in perturbation theory ${ }^{10}$. In extracting these fragmentation functions from data on $B$ hadron production in $e^{+} e^{-}$collisions, several choices are made, related to the order of perturbation theory, the incorporation of mass effects in the matrix elements, the resummation of potentially large perturbative terms, the inclusion of power corrections ${ }^{11}$, the correction of data for parton showers or the parametric form of the ansatz used in the determination. Unfortunately, the sensitivity of the fragmentation function on the assumptions used in the extraction from $e^{+} e^{-}$ spectra is often overlooked when using this fragmentation function to compute heavy hadron spectra at colliders.

Recently, an approach incorporating quark mass effects, perturbatively calculable components of the heavy quark fragmentation function ${ }^{10}$ and resummation of large logarithms up to the next-to-leading logarithmic level has been put forward with the fixed-order next-to-leading log (FONLL) scheme ${ }^{12}$. This approach requires only a small, genuinely nonperturbative component of the fragmentation function to be fitted to $e^{+} e^{-}$data. In order to expose the information content actually relevant to heavy hadron spectra at hadron colliders, this fit is done in moment space.

In view of new data from $\mathrm{ALEPH}^{13}$, a phenomenological study of $B$ hadron production at colliders based on the FONLL scheme was performed ${ }^{14}$. It was shown that the consistent treatment of the fragmentation function in extraction and prediction reduced the discrepancy between data and theoretical prediction considerably. The theoretical prediction is however still falling somewhat short of the experimental data, which is probably due to currently uncalculated corrections beyond NLO. More recently, the same framework was applied to charmed hadron production at hadron colliders ${ }^{15}$. In this case, one also observes that the experimental data ${ }^{16}$ exceed the theoretical prediction, Figure 2, although the effect is less pronounced than for bottom hadron production. Comparison of massless ${ }^{17}$ and massive ${ }^{15}$ 


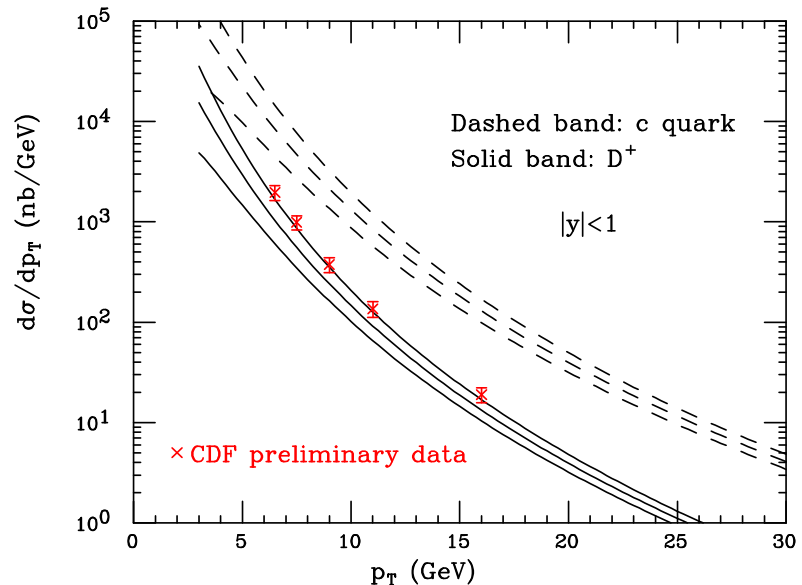

Figure 2. Transverse momentum spectrum of $D^{+}$hadrons at CDF, compared to calculation using FONLL fragmentation functions ${ }^{15}$.

calculations for hadronic charm production ${ }^{16}$ indicates only moderate mass effects, which are smaller than the theoretical uncertainty.

Many collider experiments also report an excess in the $b$ quark production spectra. In interpreting these data, it must always be kept in mind that it is not $b$ quark but $B$ hadron production which is observed in the experiment. Information on $b$ quark production is only inferred from these data using some model for the heavy quark fragmentation. As discussed above, there are numerous ambiguities, which can yield inconsistent predictions if not implemented consistently. In view of the rather sizable effects due to a consistent treatment of the fragmentation function observed on the $B$ hadron spectra at CDF, it might be that the data sets on $b$ quark spectra have to be reanalyzed incorporating the new experimental and theoretical information on the $b$ quark fragmentation functions in a consistent manner.

\section{Jets and Multiparticle Production}

Hadronic jets at large transverse momenta are produced very copiously at colliders. Final states with a small number of jets are measured to very high experimental accuracy, such that they can be used for precision measurements of the strong coupling constant and of parton distribution functions. For these measurements, the uncertainty on the theoretical prediction is often the dominant source of error, and one would consequently like to have theoretical calculations to be more accurate, which implies in general an extension towards higher perturbative orders. Multiparton final states, involving a large number of jets, can on the other hand mimic final state signatures induced by physics beyond the standard model, thus forming an irreducible background to searches. For these, QCD predictions serve as a guidance to devise search strategies, and one demands QCD to yield a description of the full hadronic final state.

\subsection{Leading order calculations}

Multiparton final states are described using leading order QCD predictions, implemented in flexible multiparton matrix element generators. These programs evaluate the scattering amplitudes using efficient representations of helicity amplitudes or fully numerically from the interaction Lagrangian. Examples of these codes are $\mathrm{VECBOS}^{18}$, COMPHEP ${ }^{19}$, MADGRAPH $^{20}$, GRACE ${ }^{21}$, HELAC $^{22}$, ALPHGEN $^{23}$ and AMEGIC $++^{24}$. Using these, the computation of $2 \rightarrow 8$ reactions is feasible on current computers. These programs are then combined with automatic integration over multiparticle phase spacs, using for example RAMBO ${ }^{25}$, PHEGAS $^{26}$ or MADEVENT $^{27}$. Most programs can be interfaced to hadronization models using standard interfaces ${ }^{28}$.

Matrix element calculations accurately include large angle single gluon radiation. At small angles from the emitting particles, one does however encounter multiple gluon radiation, which can be accounted for by parton showers. Recently, a generic procedure was devised to combine both descriptions for multiparton final states in a modified matrix element plus vetoed parton shower ${ }^{29}$.

Including these developments, leading order QCD provides the basis of Monte Carlo event generators. However, its predictions contain large (and non-quantifiable) errors due to the setting of renormalization and factorization scales. Leading order QCD is therefore good tool to estimate relative magnitudes of processes and to design searches. Once precision is required (e.g. to identify a discovery with a particular model), it is not sufficient. 
Midcone and inclusive $\mathrm{k}_{\perp}$ algorithms

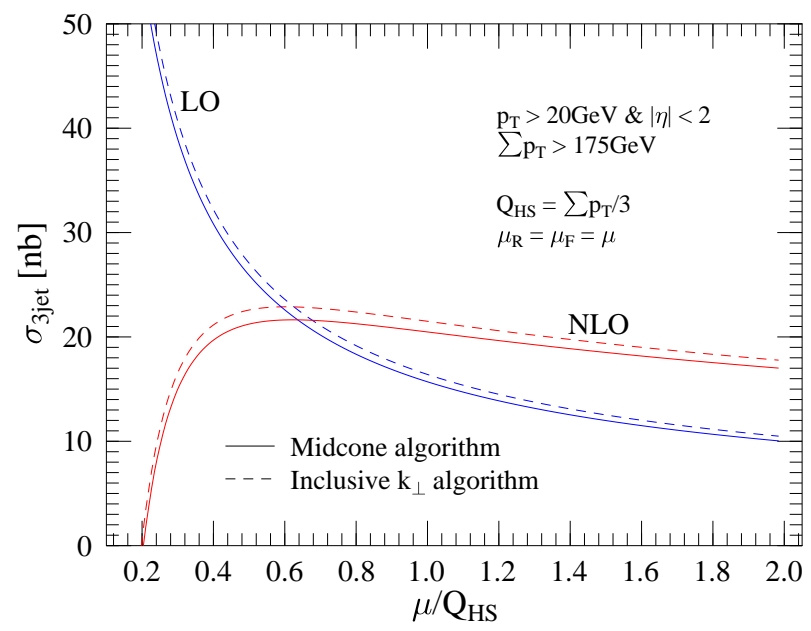

Figure 3. Dependence of the NLO prediction for the $3 j$ cross section at the Tevatron on renormalization and factorization scale $^{32}$.

\subsection{Next-to-leading order calculations}

Including next-to-leading order QCD corrections improves the theoretical predictions in numerous ways by reducing the renormalization scale uncertainty, providing reliable normalizations of cross sections, and reliable error estimates. Moreover, NLO is the first order where differences between jet algorithms show up. In contrast to leading order, there is no generic procedure for doing NLO calculations, such that each new process under consideration implies a completely new calculation.

For hadron colliders, NLO results are available for all relevant $2 \rightarrow 2$ reactions; $2 \rightarrow 3$ reactions are the current frontier. A number of $2 \rightarrow 3$ results (each involving several man-years of work) became available recently: $p p \rightarrow V+2 j^{30}$, ep $\rightarrow(3+1) j^{31}$, $p p \rightarrow 3 j^{32}, p p \rightarrow \gamma \gamma+j^{33}, p p \rightarrow t \bar{t} H^{34}$ and the vector boson fusion processes $p p \rightarrow H+2 j^{35}, p p \rightarrow V+2 j^{36}$. Some of the features of NLO calculations, such as the improved scale dependence and the differences between jet algorithms are illustrated in Figure 3.

To overcome the large amount of work required for each NLO calculation, efforts are under way towards their automatization. The NLO calculation for an $n$ parton reactions contains the one-loop $n$ parton matrix elements, the tree level $n+1$ parton matrix elements and a procedure to extract the infrared singularities from both and to combine them. While this procedure has been automatized for the

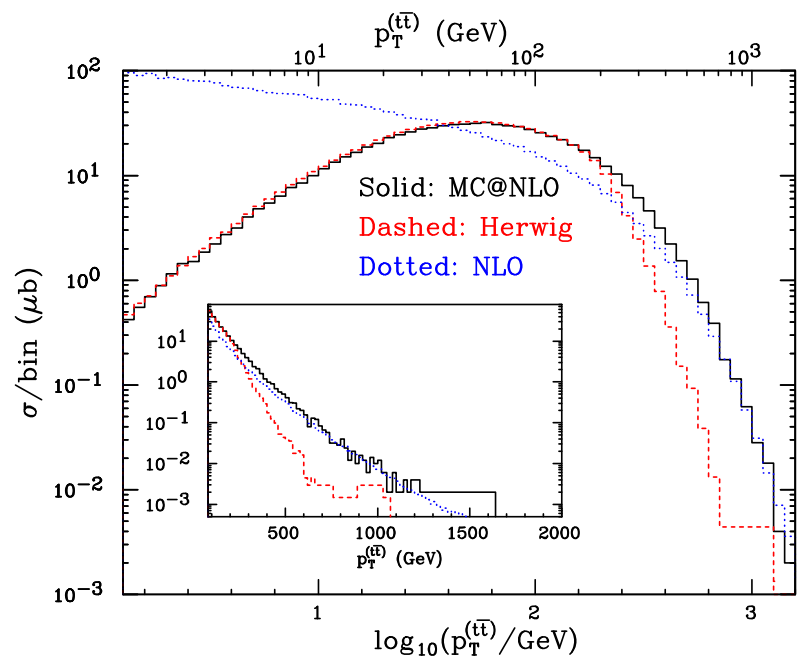

Figure 4. Transverse momentum distribution of top quark pairs at the LHC as predicted by MC@NLO ${ }^{42}$.

tree level real radiation matrix elements long ago ${ }^{37}$, there is at present no automatic procedure to compute one-loop integrals. Very recently several algorithms were proposed, including the analytic reduction of hexagon integrals ${ }^{38}$, a subtraction formalism for virtual corrections ${ }^{39}$ and the numerical evaluation of hexagon integrals ${ }^{40}$.

Another important development is the combination of NLO calculations with parton showers, as realized in the MC@NLO approach ${ }^{41}$. This approach introduces a modified NLO subtraction method, where both real and virtual contributions become initial conditions for the parton shower. In this, hard radiation is accurately described by the NLO matrix element, while multiple soft radiation is accounted for by the parton shower; a double counting of contributions is avoided. So far, this formalism has been applied to $V V, b \bar{b}$ and $t \bar{t}$ production at hadron collid-

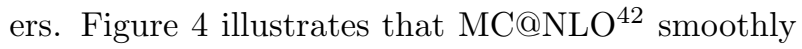
connects the kinematic region dominated by multiple radiation at small transverse momenta to the region controlled by single hard radiation at large transverse momenta.

\subsection{Next-to-next-to-leading order calculations}

Despite the evidently good agreement of NLO QCD with experimental data on jet production rates, predictions to this order are insufficient for many applications. For example, if one uses data on the single 


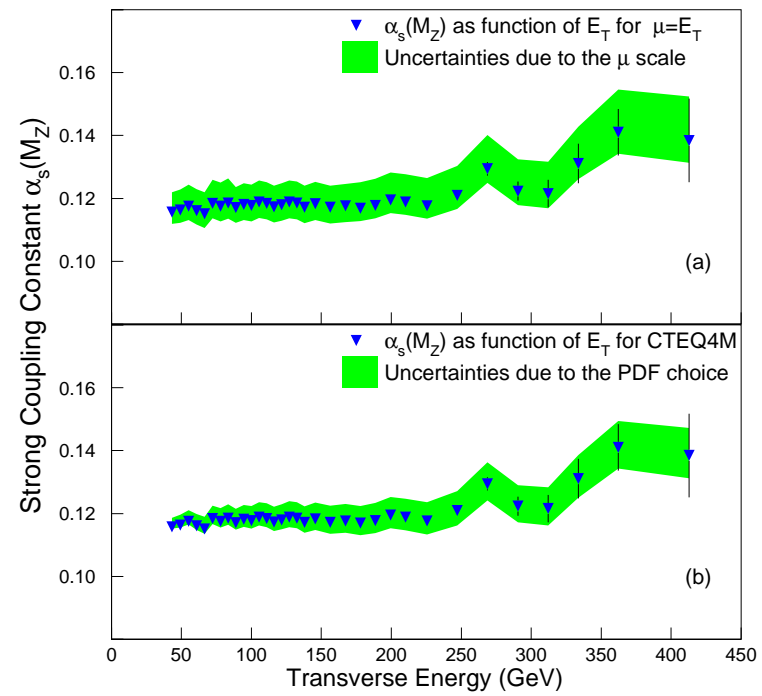

Figure 5. Errors on extraction of $\alpha_{s}$ from single jet inclusive cross section at $\mathrm{CDF}{ }^{43}$.

jet inclusive cross section ${ }^{43}$ compared to the NLO theoretical prediction ${ }^{44,45}$ to determine the strong coupling constant $\alpha_{s}$, it turns out that the dominant source of error on this extraction is due to unknown higher order corrections. Given that the theoretical prediction to infinite order in perturbation theory should be independent of the choice of renormalization and factorization scale, this error can be estimated from the variation of the extracted $\alpha_{s}$ under variation of these scales, as seen in Figure 5. As a result, CDF find from their Run I data

$$
\begin{aligned}
\alpha_{s}\left(M_{Z}\right)= & 0.1178 \pm 0.0001(\mathrm{stat})_{-0.0095}^{+0.0081}(\mathrm{sys}) \\
& { }_{-0.0047}^{+0.0001}(\mathrm{scale}) \pm 0.0059(\mathrm{pdf})
\end{aligned}
$$

It can be seen that the statistical error is already negligible; improvements in the systematic error can be anticipated in the near future. To lower the theoretical error, it is mandatory to compute next-to-nextto-leading order (NNLO) corrections to the single jet inclusive cross section.

A similar picture is true in $e^{+} e^{-}$annihilation into three jets and deep inelastic $(2+1)$ jet production, where the error on the extraction of $\alpha_{s}$ from experimentally measured jet shape observables ${ }^{46}$ is completely dominated by the theoretical uncertainty inherent in the NLO QCD calculations.

Besides lowering the theoretical error, there is a number of other reasons to go beyond NLO in the description of jet observables. While jets at NLO are modeled theoretically by at most two partons,
NNLO allows up to three partons in a single jet, thus improving the matching of experimental and theoretical jet definitions and resolving the internal jet structure. At hadron colliders, NNLO does also account for double initial state radiation, thus providing a perturbative description for the transverse momentum of the hard final state. Finally, including jet data in a global NNLO fit of parton distribution functions, one anticipates a lower error on the prediction of benchmark processes at colliders.

The calculation of jet observables at NNLO requires a number of different ingredients. To compute the corrections to an $n$-jet observable, one needs the two-loop $n$ parton matrix elements, the oneloop $n+1$ parton matrix elements and the tree level $n+2$ parton matrix elements. Since the latter two contain infrared singularities due to one or two partons becoming theoretically unresolved (soft or collinear), one needs to find one- and two-particle subtraction terms, which account for these singularities in the matrix elements, and are sufficiently simple to be integrated analytically over the unresolved phase space. One-particle subtraction at tree level is well understood from NLO calculations ${ }^{37}$ and general algorithms are available for one-particle subtraction at one loop ${ }^{47}$, in a form that could recently be integrated analytically ${ }^{48}$. Tree level two-particle subtraction terms have been extensively studied in the literature ${ }^{49}$, their integration over the unresolved phase space was up to now made only in one particular infrared subtraction scheme in the calculation of higher order corrections to the photon-plus-onejet rate in $e^{+} e^{-}$annihilation $^{50}$. The same techniques (and the same scheme) were used very recently in the rederivation of the time-like gluon-to-gluon splitting function from splitting amplitudes ${ }^{51}$. A general twoparticle subtraction procedure is still lacking at the moment, although progress on this is anticipated in the near future.

Concerning virtual two-loop corrections to jetobservables related to $2 \rightarrow 2$ scattering and $1 \rightarrow 3$ decay processes, enormous progress has been made in the past years. Much of this progress is due to several technical developments concerning the evaluation of two-loop multi-leg integrals. Using iterative algorithms ${ }^{52}$, one can reduce the large number of two-loop integrals by means of integration-by-parts ${ }^{53}$ and Lorentz invariance ${ }^{54}$ identities to a small number of master integrals. The master integrals relevant 
to two-loop jet physics are two-loop four-point functions with all legs on-shell ${ }^{55}$ or one leg off-shell ${ }^{56}$, which were computed using explicit integration or implicitly from their differential equations ${ }^{54}$.

Combing the reduction scheme with the master integrals, it is straightforward to compute the twoloop matrix elements relevant to jet observables using computer algebra ${ }^{57}$. Following this procedure, massless two-loop matrix elements were obtained for Bhabha scattering ${ }^{58}$, parton-parton scattering into two partons ${ }^{59}$, parton-parton scattering into two photons ${ }^{60}$, as well as light-by-light scattering ${ }^{61}$. Two-loop corrections were also computed for the offshell process $\gamma^{*} \rightarrow q \bar{q} g^{62}$, relevant to $e^{+} e^{-} \rightarrow 3 j$. Part of these results were already confirmed ${ }^{63}$ using an independent method ${ }^{64}$. Related to $e^{+} e^{-} \rightarrow 3 j$ by analytic continuation ${ }^{65}$ are $(2+1) j$ production in $e p$ collisions and $V+j$ production at hadron colliders. A strong check on all these two-loop results is provided by the agreement of the singularity structure with predictions obtained from an infrared factorization formula ${ }^{66}$.

More recently, first results were obtained for master integrals involving massive internal propagators, as appearing in the two-loop QED corrections to the $\gamma^{*} \rightarrow Q \bar{Q}$ vertex $^{67}$ or in the two-loop electroweak corrections to the $V \rightarrow q \bar{q}$ vertex $^{68}$.

\section{Photons}

Photons and gauge bosons provide very prominent final state signatures at colliders. Their study allows the precise determination of electroweak parameters at hadron colliders, and their final state signatures are often background to searches, such as photon pair production to the Higgs search in the lower mass range.

\subsection{Isolated Photons}

Photons produced in hadronic collisions arise essentially from two different sources: 'direct' or 'prompt' photon production via hard partonic processes such as $q g \rightarrow q \gamma$ and $q \bar{q} \rightarrow g \gamma$ or through the 'fragmentation' of a hadronic jet into a single photon carrying a large fraction of the jet energy. The former gives rise to perturbatively calculable short-distance contributions whereas the latter is primarily a long distance process which cannot be calculated pertur- batively and is described in terms of the quark-tophoton fragmentation function. In principle, this fragmentation contribution could be suppressed to a certain extent by imposing isolation cuts on the photon. Commonly used isolation cuts are defined by admitting only a maximum amount of hadronic energy in a cone of a given radius around the photon. An alternative procedure is the democratic clustering approach suggested in ${ }^{69}$, which applies standard jet clustering algorithms to events with final state photons, treating the photon like any other hadron in the clustering procedure. Isolated photons are then defined to be photons carrying more than some large, predefined amount of the jet energy.

Both types of isolation criteria infrared safe, although the matching of experimental and theoretical implementations of these criteria is in general far from trivial. It was pointed out recently ${ }^{70}$ that cone-based isolation criteria fail for small cone sizes $R$ (once $\alpha_{s} \ln R^{-2} \sim 1$ ), since the isolated photon cross section exceeds the inclusive photon cross section. This problem can only be overcome by a resummation of the large logarithms induced by the cone size parameter. The contribution from photon fragmentation to isolated photon cross sections at hadron colliders is sensitive (for both types of isolation criteria) on the photon fragmentation function at large momentum transfer, which has up to now been measured only at $\mathrm{LEP}^{71,72}$. Further information on the photon fragmentation function at large momentum transfer might be gained from yet unanalyzed LEP data or from the study of photon-plus-jet final states in deep inelastic scattering at HERA ${ }^{73}$, where first data are now becoming available ${ }^{74}$.

\subsection{Photon Pairs}

One of the most promising channels for the discovery of a light Higgs boson $\left(m_{H} \lesssim 140 \mathrm{GeV}\right)$ at the LHC is based largely on the observation of the rare decay to two photons. To perform an accurate background subtraction for this observable, one requires a precise prediction for QCD reactions yielding di-photon final states. At first sight, the $\mathcal{O}\left(\alpha_{s}^{0}\right)$ process $q \bar{q} \rightarrow \gamma \gamma$ yields the leading contribution. However, due to the large gluon luminosity at the LHC, both $q g \rightarrow q \gamma \gamma$ $\left(\mathcal{O}\left(\alpha_{s}^{1}\right)\right)$ and $g g \rightarrow \gamma \gamma\left(\mathcal{O}\left(\alpha_{s}^{2}\right)\right)$ subprocesses yield contributions of comparable magnitude. The NLO corrections to the $q \bar{q}$ and $q g$ subprocesses have been 


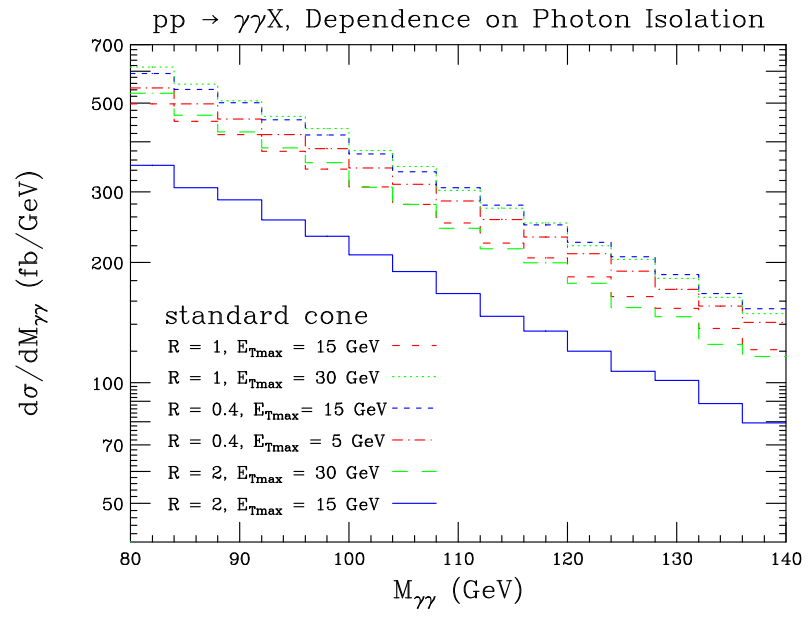

Figure 6. Photon pair production for different isolation criteria $^{76}$.

known for quite some time, these are implemented in the flexible parton level event generator DIPHOX ${ }^{75}$. Most recently, NLO corrections were also derived for the $g g$ subprocess $^{76}$. Since the lowest order contribution to this process is already mediated by a quark loop, this calculation contains some of the features appearing in jet physics only at NNLO, such as twoloop QCD amplitudes and unresolved limits of oneloop amplitudes (see Section 3.3 above). Another important new result are the NLO corrections to two-photon-plus-jet production ${ }^{33}$, forming the background to Higgs boson detection at large transverse momenta.

It must be kept in mind that the di-photon cross sections are highly sensitive on the isolation criteria applied to the photons, Figure 6, with a substantial contribution arising from photon fragmentation at large momentum transfers ${ }^{77}$. Moreover, it is experimentally difficult to distinguish photons from highly energetic neutral pions which decay into a closely collimated photon pair, mimicking a single photon signature. The pion background in photon pair production has been studied to $\mathrm{NLO}^{78}$ and implemented in DIPHOX, showing that in particular the $\pi^{0} \gamma$ channel remains comparable to the $\gamma \gamma$ channel even for tight isolation criteria.

\section{Higgs and Gauge Boson Production}

The search for the Higgs boson is one of primary goals of present and future hadron collider experi- ments, where one expects the main production channel to be gluon fusion, mediated through a top quark loop. To a good approximation ${ }^{79}$, one can use an effective gluon-gluon-Higgs coupling to describe this process in perturbative QCD (provided the leading order mass dependence is factored out explicitly). In this approximation, the calculation higher order corrections to inclusive Higgs production becomes very similar to the analogous calculation for gauge boson production.

Inclusive vector boson production has been computed to $\mathrm{NNLO}^{80}$ already more than ten years ago. Very recently, these results have been verified for the first time in an independent calculation ${ }^{81}$, carried out in the context of the derivation of NNLO corrections to inclusive Higgs boson production.

\subsection{Higgs Boson}

The NNLO corrections to the Higgs production cross section were obtained first in the soft/collinear approximation $^{82}$; shortly thereafter, the full coefficient functions were obtained by expansion around the soft limit ${ }^{81}$, and fully analytically ${ }^{83}$ by extending the IBP/LI reduction method and the differential equation technique (see Section 3.3) to compute double real emission contributions. These results were confirmed independently ${ }^{84}$ using the techniques of the original vector boson calculation. It turned out that inclusion of NNLO corrections yields a sizable enhancement of the Higgs production cross section, Figure 7, and a reduction of the uncertainty due to renormalization and factorization scale. Recently, this calculation was further improved by the inclusion of effects due to soft gluon resummation ${ }^{85}$. Further NNLO results on inclusive Higgs boson production involve pseudoscalar Higgs production through gluon fusion ${ }^{86}$, Higgs production in bottom quark fusion $^{87}$ and Higgs-strahlung off a vector boson ${ }^{88}$.

Since hadron collider experiments only cover a limited range of the final state phase space, it is very desirable to have not only predictions for the inclusive Higgs production cross sections, but also for differential distributions in rapidity and transverse momentum. Next-to-leading order corrections to both distributions ${ }^{89,90,91}$ became available recently. The rapidity distribution, Figure 8, is only moderately modified, but extends out significantly beyond the experimental coverage. The calculation of correc- 


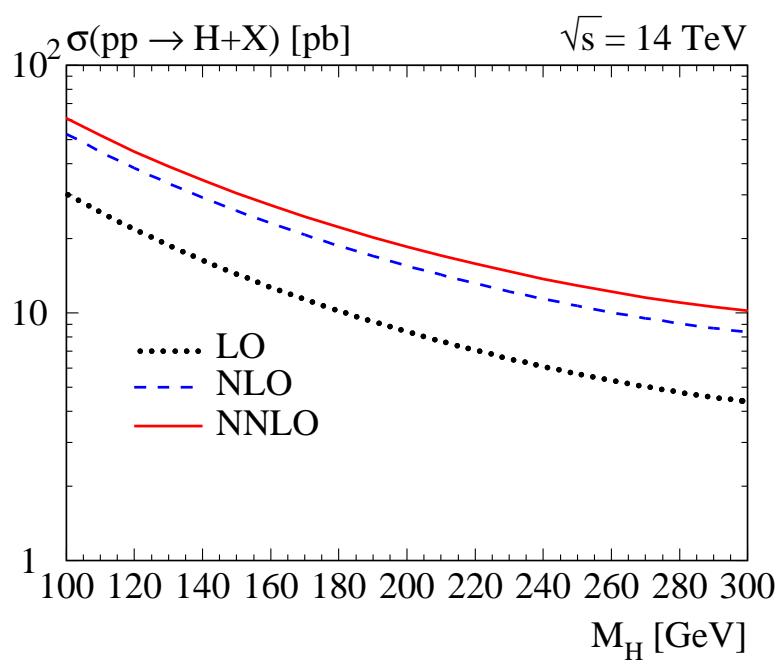

Figure 7. Inclusive Higgs production at the $\mathrm{LHC}^{81}$.

tions to transverse momentum distributions is reliable only for transverse momenta larger or equal to the Higgs boson mass, while multiple soft gluon radiation plays a crucial role at lower transverse momenta. The resummation of these corrections was performed recently ${ }^{92}$ to NNLL accuracy, Figure 9.

\subsection{Gauge Bosons}

The production cross sections for $W^{ \pm}$and $Z^{0}$ bosons at hadron colliders are well understood both experimentally and theoretically. At present, these cross sections are measured to an error of about $10 \%$ from Tevatron Run I, largely limited by statistics. A considerable reduction of the experimental error is anticipated from Run II and for the LHC. On the theory side, inclusive vector boson production has been computed to $\mathrm{NNLO}^{80,81}$.

Given the good theoretical and experimental understanding of $W^{ \pm}$and $Z^{0}$ boson production, it has been suggested to use these for a determination of the LHC luminosity ${ }^{93}$. In practice, it turns out that it is not possible to measure the fully inclusive production cross sections, but only cross sections integrated over a restricted range in rapidity, for which NNLO corrections were also computed very recently ${ }^{94}$. For the $W^{ \pm}$production, which is observed only through the $l \nu$ decay channel, it is moreover mandatory to compute the spatial distribution of the decay products, which is however only known to $\mathrm{NLO}^{45}$.

A crucial ingredient to precision NNLO predic-

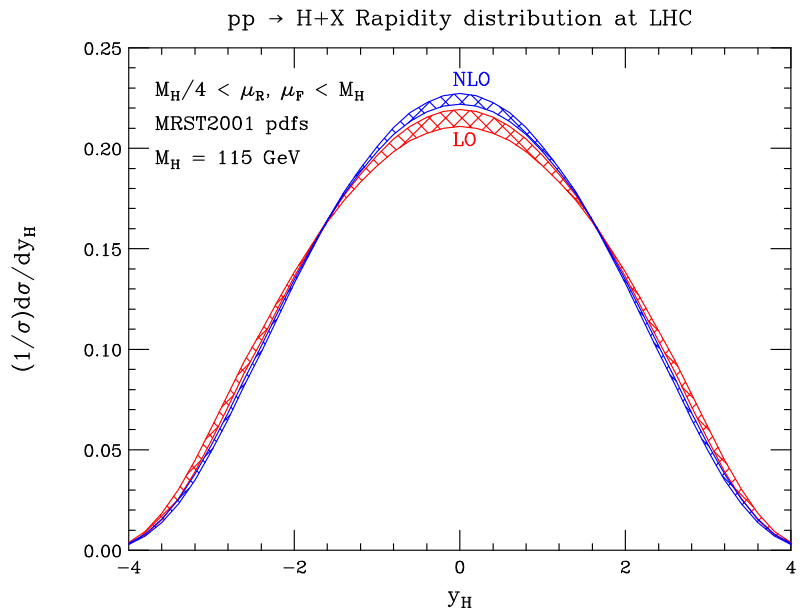

Figure 8. Rapidity distribution of Higgs bosons at $\mathrm{LHC}^{89}$.

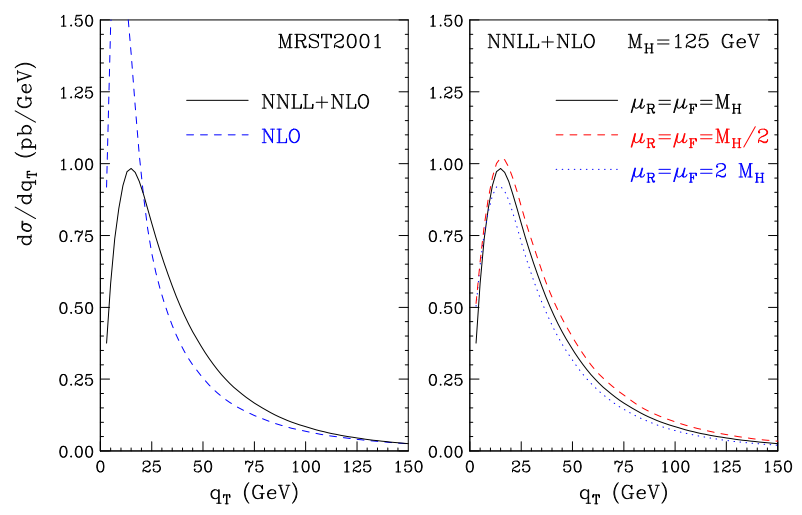

Figure 9. Transverse momentum spectrum of Higgs bosons at LHC, including soft gluon resummation ${ }^{92}$.

tions of these cross sections at LHC are parton distributions accurate to this order. The determination of parton distributions from a global fit to experimental data is described in great detail in Robert Thorne's talk at this conference ${ }^{95}$. To perform a fit at NNLO, one needs on the one hand the coefficient functions for all contributing observables (deep inelastic scattering, Drell-Yan process, jet production and possibly direct photon production) to NNLO. At present, only Drell-Yan process and deep inelastic scattering are known to this order. On the other hand, also the partonic splitting kernels (Altarelli-Parisi splitting functions) are required to NNLO accuracy. At the moment, this calculation is ongoing. The method applied in this calculation is the determination of the splitting functions from the forward photon-parton scattering amplitude at three loops, evaluated in moments of the external partonic momentum. Interme- 
diate results involve some fixed moments ${ }^{96}$, as well as all moments for the non-singlet fermionic loops ${ }^{97}$.

\section{Conclusions and Outlook}

QCD affects all observables studied at present and future hadron colliders. Given the anticipated luminosity of these machines, QCD reactions there will be precision physics, very much like electroweak physics was precision physics in the LEP era. The study of many of the standard scattering reactions will allow a precise determination of the strong coupling constant, electroweak parameters, quark masses and parton distribution functions. In turn, this information translates in improved predictions for new physics signals and their backgrounds.

Both the precision determination of standard model parameters (and auxiliary quantities) and the design of search strategies for new physics effects require substantial input from theoretical calculations. For the purpose of drafting searches for physics beyond the standard model, one often requires predictions for multiparticle final states, which are at present only available at the leading order. Such accuracy is in general sufficient for this purpose, given that the available generic programs also provide interfaces to partonic showers and hadronization models, thus predicting fully hadronic events, which can be further processed through detector simulations. Next-to-leading order calculations will be important to refine searches, and to identify potential new signals, since quantitative predictions start to become reliable only at this order. The current frontier of NLO calculations are $2 \rightarrow 3$ reactions, where some of the most prominent observables are known. Extension to $2 \rightarrow 4$ reactions will require new theoretical tools, in particular towards generic, process independent algorithms. Another important development in NLO calculations is the interface to partonic showers, which has recently been devised. For the precise extraction of standard model parameters from benchmark reactions, NNLO calculations will be mandatory, since these reactions are (already at present colliders) measured to a level of accuracy at which the theoretical error on the NLO calculation becomes the dominant source of uncertainty. Presently, first NNLO calculations were performed for $2 \rightarrow 1$ reactions, and $2 \rightarrow 2$ calculations are well under way. For applications at hadron colliders, NNLO calcula- tions become only meaningful if augmented by parton distributions accurate to NNLO, which require the knowledge on the three-loop splitting functions, which are also calculated at present.

Many observables do moreover require the resummation of large logarithms spoiling the convergence of the perturbative series. Fragmentation effects enter many observables with identified particles in the final state. In particular, a consistent treatment of heavy quark fragmentation effects can account for a large part of the observed discrepancy in $B$ hadron spectra, and quark-to-photon as well as quark-to-pion fragmentation yield important contributions to photon pair final states forming an important background to Higgs searches. Much valuable information on these fragmentation functions is contained in data from LEP, and should be extracted (as long as this is still a feasible task) to improve predictions for collider observables.

\section{References}

1. P. Nason, S. Dawson and R. K. Ellis, Nucl. Phys. B 303 (1988) 607; W. Beenakker, W. L. van Neerven, R. Meng, G. A. Schuler and J. Smith, Nucl. Phys. B 351 (1991) 507.

2. R. Bonciani, S. Catani, M. L. Mangano and P. Nason, Nucl. Phys. B 529 (1998) 424 [arXiv:hepph/9801375]; arXiv:hep-ph/0307035.

3. N. Kidonakis, E. Laenen, S. Moch and R. Vogt, Phys. Rev. D 64 (2001) 114001 [arXiv:hepph/0105041].

4. T. Affolder et al. [CDF Collaboration], Phys. Rev. D 64 (2001) 032002 [arXiv:hep-ex/0101036]; V. M. Abazov et al. [D0 Collaboration], Phys. Rev. D 67 (2003) 012004 [arXiv:hep-ex/0205019].

5. I. Abt et al. [HERA-B Collaboration], Eur. Phys. J. C 26 (2003) 345 [arXiv:hep-ex/0205106].

6. M. Cacciari, S. Frixione, M. L. Mangano, P. Nason and G. Ridolfi, arXiv:hep-ph/0303085.

7. D. Acosta et al. [CDF Collaboration], Phys. Rev. D 65 (2002) 052005 [arXiv:hep-ph/0111359].

8. B. Abbott et al. [D0 Collaboration], Phys. Rev. Lett. 85 (2000) 5068 [arXiv:hep-ex/0008021].

9. S. Frixione and M.L. Mangano, Nucl. Phys. B 483 (1997) 321 [arXiv:hep-ph/9605270].

10. B. Mele and P. Nason, Nucl. Phys. B 361 (1991) 626.

11. M. Cacciari and E. Gardi, Nucl. Phys. B 664 (2003) 299 [arXiv:hep-ph/0301047].

12. M. Cacciari, M. Greco and P. Nason, JHEP 9805 (1998) 007 [arXiv:hep-ph/9803400].

13. A. Heister et al. [ALEPH Collaboration], Phys. Lett. B 512 (2001) 30 [arXiv:hep-ex/0106051]. 
14. M. Cacciari and P. Nason, Phys. Rev. Lett. 89 (2002) 122003 [arXiv:hep-ph/0204025].

15. M. Cacciari and P. Nason, JHEP 0309 (2003) 006 [arXiv:hep-ph/0306212].

16. D. Acosta et al. [CDF Collaboration], arXiv:hepex/0307080.

17. B. A. Kniehl, G. Kramer and B. Pötter, Nucl. Phys. B 597 (2001) 337 [arXiv:hep-ph/0011155].

18. F. A. Berends, H. Kuijf, B. Tausk and W. T. Giele, Nucl. Phys. B 357 (1991) 32.

19. A. Pukhov et al., arXiv:hep-ph/9908288.

20. T. Stelzer and W. F. Long, Comput. Phys. Commun. 81 (1994) 357 [arXiv:hep-ph/9401258].

21. F. Yuasa et al., Prog. Theor. Phys. Suppl. 138 (2000) 18 [arXiv:hep-ph/0007053].

22. A. Kanaki and C. G. Papadopoulos, Comput. Phys. Commun. 132 (2000) 306 [arXiv:hep-ph/0002082].

23. M. L. Mangano, M. Moretti, F. Piccinini, R. Pittau and A. D. Polosa, JHEP 0307 (2003) 001 [arXiv:hepph/0206293].

24. F. Krauss, R. Kuhn and G. Soff, JHEP 0202 (2002) 044 [arXiv:hep-ph/0109036].

25. R. Kleiss, W. J. Stirling and S. D. Ellis, Comput. Phys. Commun. 40 (1986) 359.

26. C. G. Papadopoulos, Comput. Phys. Commun. 137 (2001) 247 [arXiv:hep-ph/0007335].

27. F. Maltoni and T. Stelzer, JHEP 0302 (2003) 027 [arXiv:hep-ph/0208156].

28. W. Giele et al., "The QCD/SM working group: Summary report,", Proceedings of Les Houches Workshop on "Physics at TeV Colliders", 2001, arXiv:hepph/0204316.

29. S. Catani, F. Krauss, R. Kuhn and B. R. Webber, JHEP 0111 (2001) 063 [arXiv:hep-ph/0109231].

30. J. Campbell and R.K. Ellis, Phys. Rev. D 65 (2002) 113007 [arXiv:hep-ph/0202176].

31. Z. Nagy and Z. Trocsanyi, Phys. Rev. Lett. 87 (2001) 082001 [arXiv:hep-ph/0104315].

32. Z. Nagy, Phys. Rev. Lett. 88 (2002) 122003 [arXiv:hep-ph/0110315]; arXiv:hep-ph/0307268.

33. V. Del Duca, F. Maltoni, Z. Nagy and Z. Trocsanyi, JHEP 0304 (2003) 059 [arXiv:hep-ph/0303012].

34. W. Beenakker, S. Dittmaier, M. Krämer, B. Plümper, M. Spira and P.M. Zerwas, Phys. Rev. Lett. 87 (2001) 201805 [arXiv:hep-ph/0107081]; Nucl. Phys. B 653 (2003) 151 [arXiv:hepph/0211352]; L. Reina and S. Dawson, Phys. Rev. Lett. 87 (2001) 201804 [arXiv:hep-ph/0107101]; S. Dawson, C. Jackson, L. H. Orr, L. Reina and D. Wackeroth, Phys. Rev. D 68 (2003) 034022 [arXiv:hep-ph/0305087].

35. T. Figy, C. Oleari and D. Zeppenfeld, arXiv:hep$\mathrm{ph} / 0306109$.

36. C. Oleari and D. Zeppenfeld, arXiv:hep-ph/0310156.

37. W.T. Giele and E.W.N. Glover, Phys. Rev. D 46 (1992) 1980; S. Catani and M. H. Seymour, Nucl. Phys. B 485 (1997) 291 [Erratum-ibid. B 510 (1997) 503] [arXiv:hep-ph/9605323].
38. T. Binoth, J. P. Guillet, G. Heinrich and C. Schubert, Nucl. Phys. B 615 (2001) 385 [arXiv:hep$\mathrm{ph} / 0106243]$

39. Z. Nagy and D. E. Soper, JHEP 0309 (2003) 055 [arXiv:hep-ph/0308127].

40. T. Binoth, G. Heinrich and N. Kauer, Nucl. Phys. B 654 (2003) 277 [arXiv:hep-ph/0210023].

41. S. Frixione and B. R. Webber, JHEP 0206 (2002) 029 [arXiv:hep-ph/0204244]; arXiv:hep-ph/0207182; arXiv:hep-ph/0307146; arXiv:hep-ph/0309186.

42. S. Frixione, P. Nason and B. R. Webber, JHEP 0308 (2003) 007 [arXiv:hep-ph/0305252].

43. T. Affolder et al. [CDF Collaboration], Phys. Rev. Lett. 88 (2002) 042001 [arXiv:hep-ex/0108034].

44. Z. Kunszt and D. E. Soper, Phys. Rev. D 46 (1992) 192; S. D. Ellis, Z. Kunszt and D. E. Soper, Phys. Rev. Lett. 69 (1992) 3615 [arXiv:hep-ph/9208249].

45. W.T. Giele, E.W.N. Glover and D.A. Kosower, Nucl. Phys. B 403 (1993) 633 [arXiv:hep-ph/9302225].

46. R. Hirosky, these proceedings.

47. Z. Bern, L.J. Dixon, D.C. Dunbar and D.A. Kosower, Nucl. Phys. B 425 (1994) 217 [arXiv:hepph/9403226]; D.A. Kosower, Nucl. Phys. B 552 (1999) 319 [arXiv:hep-ph/9901201]; D.A. Kosower and P. Uwer, Nucl. Phys. B 563 (1999) 477 [arXiv:hep-ph/9903515]; Z. Bern, V. Del Duca and C.R. Schmidt, Phys. Lett. B 445 (1998) 168 [arXiv:hep-ph/9810409]; Z. Bern, V. Del Duca, W.B. Kilgore and C.R. Schmidt, Phys. Rev. D 60 (1999) 116001 [arXiv:hep-ph/9903516]; S. Catani and M. Grazzini, Nucl. Phys. B 591 (2000) 435 [arXiv:hep$\mathrm{ph} / 0007142]$.

48. D. A. Kosower, Phys. Rev. Lett. 91 (2003) 061602 [arXiv:hep-ph/0301069]; S. Weinzierl, JHEP 0307 (2003) 052 [arXiv:hep-ph/0306248].

49. J.M. Campbell and E.W.N. Glover, Nucl. Phys. B 527 (1998) 264 [arXiv:hep-ph/9710255]; S. Catani and M. Grazzini, Phys. Lett. B 446 (1999) 143 [arXiv:hep-ph/9810389]; Nucl. Phys. B $\mathbf{5 7 0}$ (2000) 287 [arXiv:hep-ph/9908523]; F.A. Berends and W.T. Giele, Nucl. Phys. B 313 (1989) 595; D. A. Kosower, Phys. Rev. D 67 (2003) 116003 [arXiv:hep-ph/0212097]; S. Weinzierl, JHEP 0303 (2003) 062 [arXiv:hep-ph/0302180].

50. A. Gehrmann-De Ridder, T. Gehrmann and E.W.N. Glover, Phys. Lett. B 414 (1997) 354 [arXiv:hep-ph/9705305]; A. Gehrmann-De Ridder and E.W.N. Glover, Nucl. Phys. B 517 (1998) 269 [arXiv:hep-ph/9707224].

51. D.A. Kosower and P. Uwer, arXiv:hep-ph/0307031.

52. S. Laporta, Int. J. Mod. Phys. A 15 (2000) 5087 [arXiv:hep-ph/0102033].

53. F.V. Tkachov, Phys. Lett. 100B (1981) 65; K.G. Chetyrkin and F.V. Tkachov, Nucl. Phys. B 192 (1981) 159.

54. T. Gehrmann and E. Remiddi, Nucl. Phys. B 580 (2000) 485 [arXiv:hep-ph/9912329].

55. V.A. Smirnov, Phys. Lett. B 460 (1999) 397 
[arXiv:hep-ph/9905323]; V.A. Smirnov and O.L. Veretin, Nucl. Phys. B 566 (2000) 469 [arXiv:hepph/9907385]; J.B. Tausk, Phys. Lett. B 469 (1999) 225 [arXiv:hep-ph/9909506]; C. Anastasiou, T. Gehrmann, C. Oleari, E. Remiddi and J.B. Tausk, Nucl. Phys. B 580 (2000) 577 [arXiv:hepph/0003261]; T. Gehrmann and E. Remiddi, Nucl. Phys. B (Proc. Suppl.) 89 (2000) 251 [arXiv:hepph/0005232]; C. Anastasiou, J.B. Tausk and M.E. Tejeda-Yeomans, Nucl. Phys. B (Proc. Suppl.) 89 (2000) 262 [arXiv:hep-ph/0005328].

56. T. Gehrmann and E. Remiddi, Nucl. Phys. B 601 (2001) 248 [arXiv:hep-ph/0008287] and 601 (2001) 287 [arXiv:hep-ph/0101124].

57. T. Gehrmann, Nucl. Phys. Proc. Suppl. 116 (2003) 13 [arXiv:hep-ph/0210157].

58. Z. Bern, L. Dixon and A. Ghinculov, Phys. Rev. D 63 (2001) 053007 [arXiv:hep-ph/0010075].

59. C. Anastasiou, E.W.N. Glover, C. Oleari and M.E. Tejeda-Yeomans, Nucl. Phys. B 601 (2001) 318 [arXiv:hep-ph/0010212]; $601 \quad$ (2001) 347 [arXiv:hep-ph/0011094]; 605 (2001) 486 [arXiv:hepph/0101304]; E.W.N. Glover, C. Oleari and M.E. Tejeda-Yeomans, Nucl. Phys. 605 (2001) 467 [arXiv:hep-ph/0102201]; E.W.N. Glover and M.E. Tejeda-Yeomans, JHEP 0306 (2003) 033 [arXiv:hep-ph/0304169]; Z. Bern, A. De Freitas and L. Dixon, JHEP 0203 (2002) 018 [arXiv:hepph/0201161]; Z. Bern, A. De Freitas and L. Dixon, JHEP 0306 (2003) 028 [arXiv:hep-ph/0304168].

60. Z. Bern, A. De Freitas and L. J. Dixon, JHEP 0109 (2001) 037 [arXiv:hep-ph/0109078]; C. Anastasiou, E.W.N. Glover and M.E. Tejeda-Yeomans, Nucl. Phys. B 629 (2002) 255 [arXiv:hep-ph/0201274].

61. Z. Bern, A. De Freitas, L.J. Dixon, A. Ghinculov and H.L. Wong, JHEP 0111 (2001) 031 [arXiv:hepph/0109079]; T. Binoth, E.W.N. Glover, P. Marquard and J.J. van der Bij, JHEP 0205 (2002) 060 [arXiv:hep-ph/0202266].

62. L.W. Garland, T. Gehrmann, E.W.N. Glover, A. Koukoutsakis and E. Remiddi, Nucl. Phys. B 627 (2002) 107 [arXiv:hep-ph/0112081] and 642 (2002) 227 [arXiv:hep-ph/0206067].

63. S. Moch, P. Uwer and S. Weinzierl, Phys. Rev. D 66 (2002) 114001 [arXiv:hep-ph/0207043].

64. S. Moch, P. Uwer and S. Weinzierl, J. Math. Phys. 43 (2002) 3363 [arXiv:hep-ph/0110083]; S. Weinzierl, Comput. Phys. Commun. 145 (2002) 357 [arXiv:math-ph/0201011].

65. T. Gehrmann and E. Remiddi, Nucl. Phys. B 640 (2002) 379 [arXiv:hep-ph/0207020].

66. S. Catani, Phys. Lett. B 427 (1998) 161 [arXiv:hepph/9802439]; G. Sterman and M. E. TejedaYeomans, Phys. Lett. B 552 (2003) 48 [arXiv:hep$\mathrm{ph} / 0210130]$.

67. R. Bonciani, P. Mastrolia and E. Remiddi, Nucl. Phys. B 661 (2003) 289 [arXiv:hep-ph/0301170]; arXiv:hep-ph/0307295.
68. U. Aglietti and R. Bonciani, Nucl. Phys. B 668 (2003) 3 [arXiv:hep-ph/0304028].

69. E.W.N. Glover and A.G. Morgan, Z. Phys. C 62 (1994) 311.

70. S. Catani, M. Fontannaz, J. P. Guillet and E. Pilon, JHEP 0205 (2002) 028 [arXiv:hep-ph/0204023].

71. D. Buskulic et al. [ALEPH Collaboration], Z. Phys. C 69 (1996) 365.

72. K. Ackerstaff et al. [OPAL Collaboration], Eur. Phys. J. C 2 (1998) 39 [arXiv:hep-ex/9708020].

73. A. Gehrmann-De Ridder, G. Kramer and H. Spiesberger, Nucl. Phys. B 578 (2000) 326 [arXiv:hepph/0003082].

74. R. Lemrani [H1 Collaboration], arXiv:hepex/0308066.

75. T. Binoth, J.P. Guillet, E. Pilon and M. Werlen, Eur. Phys. J. C 16 (2000) 311 [arXiv:hep-ph/9911340].

76. Z. Bern, L. Dixon and C. Schmidt, Phys. Rev. D 66 (2002) 074018 [arXiv:hep-ph/0206194].

77. T. Binoth, J. P. Guillet, E. Pilon and M. Werlen, Phys. Rev. D 63 (2001) 114016 [arXiv:hep$\mathrm{ph} / 0012191]$

78. T. Binoth, J. P. Guillet, E. Pilon and M. Werlen, Eur. Phys. J. C 24 (2002) 245 [arXiv:hep$\mathrm{ph} / 0111043]$.

79. M. Spira, A. Djouadi, D. Graudenz and P. M. Zerwas, Nucl. Phys. B 453 (1995) 17 [arXiv:hepph/9504378]; M. Spira, Fortsch. Phys. 46 (1998) 203 [arXiv:hep-ph/9705337].

80. R. Hamberg, W. L. van Neerven and T. Matsuura, Nucl. Phys. B 359 (1991) 343 [Erratum-ibid. B 644 (2002) 403].

81. R.V. Harlander and W.B. Kilgore, Phys. Rev. Lett. 88 (2002) 201801 [arXiv:hep-ph/0201206].

82. R.V. Harlander, Phys. Lett. B 492 (2000) 74 [arXiv:hep-ph/0007289]; S. Catani, D. de Florian and M. Grazzini, JHEP 0105 (2001) 025 [arXiv:hepph/0102227]; R.V. Harlander and W.B. Kilgore, Phys. Rev. D 64 (2001) 013015 [arXiv:hep$\mathrm{ph} / 0102241]$

83. C. Anastasiou and K. Melnikov, Nucl. Phys. B 646 (2002) 220 [arXiv:hep-ph/0207004].

84. V. Ravindran, J. Smith and W. L. van Neerven, Nucl. Phys. B 665 (2003) 325 [arXiv:hepph/0302135].

85. S. Catani, D. de Florian, M. Grazzini and P. Nason, JHEP 0307 (2003) 028 [arXiv:hep-ph/0306211].

86. R.V. Harlander and W.B. Kilgore, JHEP 0210 (2002) 017 [arXiv:hep-ph/0208096]; C. Anastasiou and K. Melnikov, arXiv:hep-ph/0208115.

87. R. V. Harlander and W. B. Kilgore, Phys. Rev. D 68 (2003) 013001 [arXiv:hep-ph/0304035].

88. O. Brein, A. Djouadi and R. Harlander, arXiv:hep$\mathrm{ph} / 0307206$.

89. C. Anastasiou, L. Dixon and K. Melnikov, Nucl. Phys. Proc. Suppl. 116 (2003) 193 [arXiv:hep$\mathrm{ph} / 0211141]$

90. D. de Florian, M. Grazzini and Z. Kunszt, Phys. 
Rev. Lett. 82 (1999) 5209 [arXiv:hep-ph/9902483].

91. V. Ravindran, J. Smith and W. L. Van Neerven, Nucl. Phys. B 634 (2002) 247 [arXiv:hepph/0201114]; C. J. Glosser and C. R. Schmidt, JHEP 0212 (2002) 016 [arXiv:hep-ph/0209248].

92. G. Bozzi, S. Catani, D. de Florian and M. Grazzini, Phys. Lett. B 564 (2003) 65 [arXiv:hep-ph/0302104].

93. A.D. Martin, R.G. Roberts, W.J. Stirling and R.S. Thorne, Eur. Phys. J. C 14 (2000) 133 [arXiv:hep-ph/9907231].

94. C. Anastasiou, L. Dixon, K. Melnikov and F. Petriello, arXiv:hep-ph/0306192.

95. R. Thorne, these proceedings, arXiv:hep$\mathrm{ph} / 0309343$.

96. S. A. Larin, P. Nogueira, T. van Ritbergen and J. A. Vermaseren, Nucl. Phys. B 492 (1997) 338 [arXiv:hep-ph/9605317]; A. Retey and J. A. Vermaseren, Nucl. Phys. B 604 (2001) 281 [arXiv:hep$\mathrm{ph} / 0007294]$.

97. S. Moch, J. A. Vermaseren and A. Vogt, Nucl. Phys. B 646 (2002) 181 [arXiv:hep-ph/0209100].

\section{DISCUSSION}

Sungwon Lee (Texas A \& M University): Currently there are large discrepancies between data and NLO QCD predictions for direct photon production. Has there been any theoretical progress on the intrinsic (effective parton) $k_{t}$ issue for direct photon production?

Thomas Gehrmann: Understanding of $k_{t}$ effects is one motivation for doing NNLO calculations. At NNLO, for the first time, we start to fully model $k_{t}$ effects due to hard parton emission in the initial state because we allow for either double emission of one of the incoming legs, or for double uncorrelated emission. So, once you have NNLO calculations for $2 \rightarrow 2$ scattering processes available, you will really have a theoretical tool for computing $k_{t}$ effects from perturbation theory.

Ikaros Bigi (Notre Dame University): You showed this very instructive curve about $b$ quark fragmentation, where you showed that the Peterson et al. prediction is less than optimal. Do you have (or does someone have) a similar curve for charm fragmentation?

Thomas Gehrmann: The recent work by Cacciari and Nason addresses this issue. They refit charm fragmentation functions using a new parameterization in moment space trying to expose the information content of LEP data relevant to proton-antiproton colliders. Comparison with old parameterizations is however not made. The basic message is that when you start fitting fragmentation functions, you have to be extremely careful that you are not introducing artifacts from the choice of parameterization which then, although giving a you a least $\chi^{2}$ fit, do not really reproduce the data because you are starting with too stiff an initial form. 